\title{
Pathway-based drug repositioning using causal inference
}

\author{
Jiao $\mathrm{Li}^{1}$, Zhiyong $\mathrm{Lu}^{2^{*}}$ \\ From Asia Pacific Bioinformatics Network (APBioNet) Twelfth International Conference on Bioinformatics \\ (InCoB2013) \\ Taicang, China. 20-22 September 2013
}

\begin{abstract}
Background: Recent in vivo studies showed new hopes of drug repositioning through causality inference from drugs to disease. Inspired by their success, here we present an in silico method for building a causal network (CauseNet) between drugs and diseases, in an attempt to systematically identify new therapeutic uses of existing drugs.

Methods: Unlike the traditional 'one drug-one target-one disease' causal model, we simultaneously consider all possible causal chains connecting drugs to diseases via target- and gene-involved pathways based on rich information in several expert-curated knowledge-bases. With statistical learning, our method estimates transition likelihood of each causal chain in the network based on known drug-disease treatment associations (e.g. bexarotene treats skin cancer).

Results: To demonstrate its validity, our method showed high performance (AUC $=0.859$ ) in cross validation. Moreover, our top scored prediction results are highly enriched in literature and clinical trials. As a showcase of its utility, we show several drugs for potential re-use in Crohn's Disease.

Conclusions: We successfully developed a computational method for discovering new uses of existing drugs based on casual inference in a layered drug-target-pathway-gene- disease network. The results showed that our proposed method enables hypothesis generation from public accessible biological data for drug repositioning.
\end{abstract}

\section{Background}

Despite the fast growth in drug research and development (R\&D) such as chemical genomics technologies [1,2] and chemical libraries [3,4], the pharmaceutical R\&D outputnew drugs brought to market-has significantly declined in recent decades. As reported in the most recent analysis, the number of new drugs approved per billion US dollars spent has halved approximately every 9 years since 1950 [5]. Discovering new uses for existing drugs, also known as drug repositioning, provides one possible solution to such a problem. The fact that existing drugs have already passed through development stages such as target validation and ADMET (absorption, distribution, metabolism, excretion and toxicity) characteristics analysis should

\footnotetext{
* Correspondence: zhiyong.lu@nih.gov

${ }^{2}$ National Center for Biotechnology Information (NCBI), National Institutes of Health, Bethesda, USA

Full list of author information is available at the end of the article
}

greatly help reduce time and risk when attempting to identify their new indications [6].

The traditional one drug-one target-one disease drug discovery model has been argued to more likely result in poor efficacy or unanticipated side effects by not taking into account the complexity of underlying mechanism $[7,8]$. Due to such limitations, network-based computational approaches were proposed recently, providing a new framework for identifying drug-repositioning opportunities. Keiser et al. predicted new targets for known drugs using drug chemical structures and their canonical biological targets, and the resulting novel drug-target network further connected drugs to new indications [9]. Li et al. measured drug pairwise similarity by combining similarity of drug chemical structures, similarity of target profiles, and interaction between target proteins [10]. Iorio et al. constructed a drug-drug similarity network using transcriptional responses (i.e., gene expression profiles)

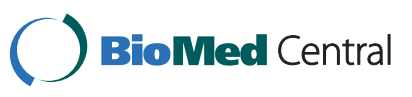


following drug treatment [11]. Recent studies [12-14] compared the drug vs. disease gene expression profiles for identifying novel treatment relationships between drugs and diseases. Other kinds of network-based approaches for drug repositioning included literature mining [15] and shared pathway analysis [16].

Different from the aforementioned computational approaches, several recent studies demonstrated the feasibility of drug repositioning through manual analysis of causal associations in drug-involved pathways [17-20]. For example, Cramer et al. found that FDA approved anticancer drug bexarotene could be potentially used for Alzheimer's Disease (AD) treatment [19] based on molecular pathway examination and analysis. More specifically, they found bexarotene activates nuclear receptors PPAR (peroxisome proliferator-activated receptor) and LXR (liver $\times$ receptor) in coordination with RXR (retinoid $\times$ receptor), thus up-regulating the expression of the ApoE (apoliporrotein E) gene. This process facilitates the clearance of $A \beta$ ( $\beta$-amyloid) from the brain, resulting in the alleviation of $\mathrm{AD}$. In this example, the chain of causality between one drug and one disease was examined and inferred by domain experts who took advantage of the following knowledge in bexarotenerelated pathways: (1) drug-target (e.g., bexarotene is an RXR agonist); (2) target involved pathway (e.g., LXR:RXR activation pathway); (3) transcriptional responses in a given pathway (e.g., increased ApoE gene expression in the LXR:RXR activation pathway); (4) genetic mechanism of disease (e.g., ApoE is associated with AD).

Motivated by the success of manual pathway analysis for drug repositioning, we developed a new computational method for building a network of causal chains between drugs and diseases, allowing for computational drug repositioning. By taking advantage of the increasing amount of expert-curated biological knowledge in the public domain (e.g. pathway information in Pathway Commons [21]), we built a multi-layer causal network (CauseNet) consisting of chains from drug to target, target to pathway, pathway to downstream gene, and gene to disease. Furthermore, we used a statistical method to learn the transition likelihood of each causal chain in the network based on those known drug-disease treatment relationships. In the prediction stage, we identified novel drug re-uses using maximum likelihood estimation. Unlike the traditional causal chain models that relied on human examination of one drug target, pathway and gene at a time, our computational model allows us to investigate all possible causal links when connecting drugs to diseases at once. To our best knowledge, this is also the first attempt of using network-based causal inference in computational drug repositioning.

\section{Methods}

In Figure 1, we show a model of our proposed CauseNet which puts causal chains from drugs to diseases in a layered network. The nodes of CauseNet are organized in five layers: drug $D\left\{d_{1}, \ldots d_{x}\right\}$, target $T\left\{t_{1}, \ldots t_{m}\right\}$, pathway $P\left\{p_{1}, \ldots p_{n}\right\}$, downstream genes $G\left\{g_{1}, \ldots, g_{k}\right\}$, and disease $S$ $\left\{s_{1}, \ldots s_{y}\right\}$. Accordingly, from top to bottom the causal links between two layers represent (1) drug $d$ acts on target $t$; (2) target $t$ participants in pathway $p$; (3) pathway $p$ affects the expression of downstream gene $g$; and (4) gene $g$ is associated with disease $s$. To construct such a network, we integrated data from heterogeneous resources which contain expert-curated knowledge of relationships between drugs, molecules and diseases. Furthermore, we learn the transition weight for each causal link in the CauseNet to distinguish the likelihood of transitions between nodes based on the known treatment relationships between drugs and diseases (details in Section computing transition weights). For instance, if drug $d_{1}$ is known to treat disease $s_{y}$, then the transition weights of the gold-colored links in Figure 1 should be promoted accordingly.

\section{Constructing CauseNet}

For constructing CauseNet, we extracted approved drugs and their targets from DrugBank [22], target-involvedpathways from Pathway Commons [21] and KEGG [23], downstream genes from Pathway Commons, and diseases and their associated genes from Comparative Toxicogenomics Database (CTD) [24]. Also from CTD, we assembled pairs of known drug-disease treatment relationships. Note that each pathway can mention information on a series of biological events such as biochemical reactions, physical interactions, transcriptional responses, and phosphorylation and enzyme catalysis. In this study, we focused on transcriptional responses (i.e., up/down regulated expression of downstream genes) in a pathway.

\section{Computing transition weights}

We represent the constructed CauseNet as a directed graph $G(V, E)$. The node set, $V(G)=\{D, T, P, G, S\}$, consists of five types of objects (i.e., drug $D$, $\operatorname{target} T$, pathway $P$, downstream gene $G$ and disease $S$ ). The edge set is denoted as $E(G) \subseteq\{D \times T, T \times P, P \times G, G \times S\}$. A complete causal chain, $c=\langle d, t, p, g, s\rangle$, represents a 4-step path from drug $d(d L D)$ to disease $\mathrm{s}(s L S)$ with a set of individual chains $E(c)=\{(d, t),(t, p),(p, g),(g, s)\} \subset E(G)$. All possible causal chains from drugs to diseases become the complete chain set $C$. We further use a subset of (treatment-enriched) chains $C^{*}\left(\right.$ i.e., $C^{*} \subset C$ ) to represent the links between drug-disease pairs of known treatment relationships. For example, as shown in Figure 1, drug $d_{1}$ is linked to diseases $s_{2}$ and $s_{y}$ through two separatechains 


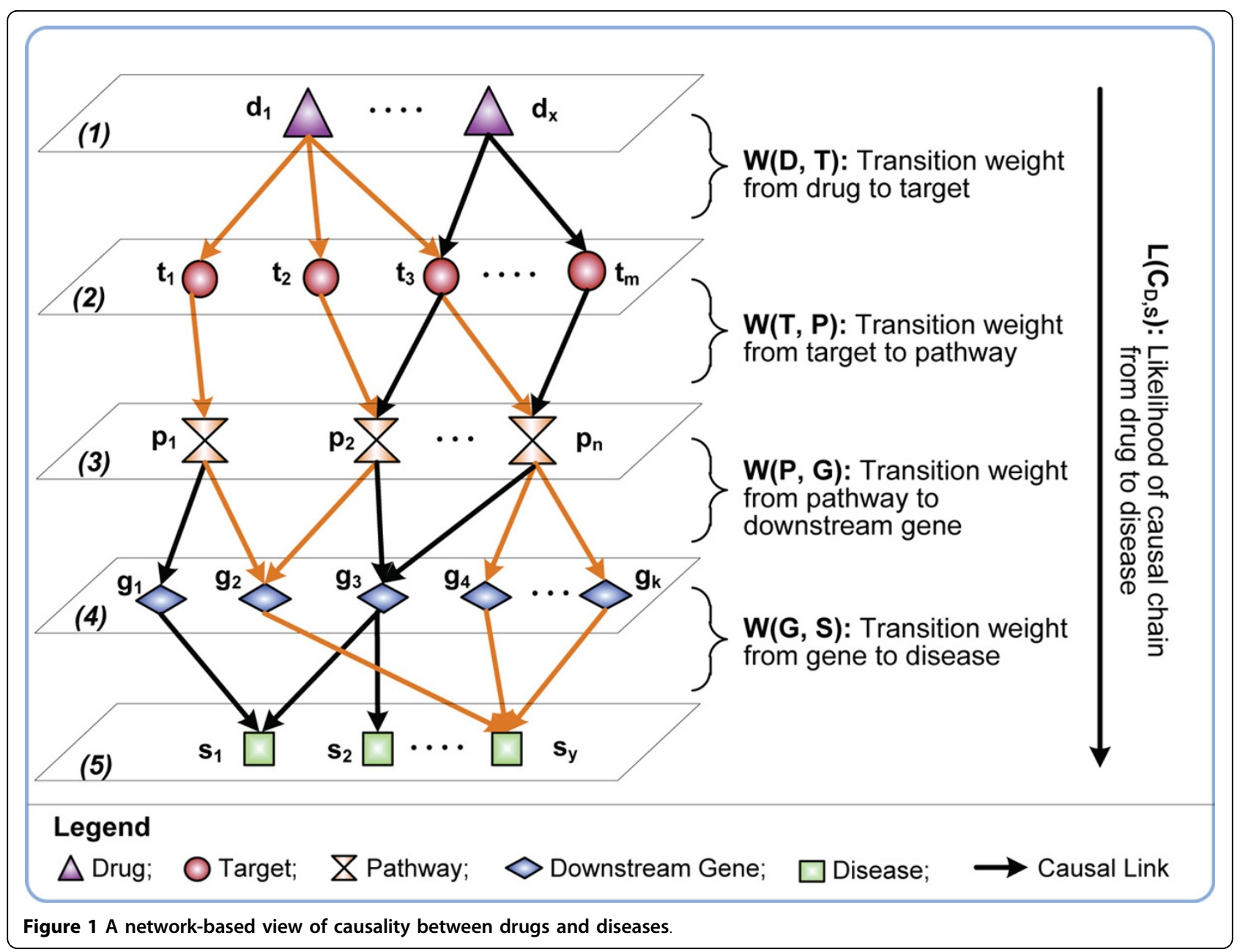

$c_{1}=<d_{1}, t_{2}, p_{2}, g_{3}, s_{2}>$ and $c_{2}=<d_{1}, t_{2}, p_{2}, g_{2}, s_{y}>$, where $c_{1}$, $c_{2} C$ and $c_{2} C^{*}\left(d_{1}\right.$ is known to treat $s_{y}$ but not $\left.s_{2}\right)$.

The graphs of the respective complete and enriched chain sets $C$ and $C^{*}$ are denoted as $G(C)=G(V(C), E(C))$ and $G\left(C^{*}\right)=G\left(V\left(C^{*}\right), E\left(C^{*}\right)\right)$, where $V\left(C^{*}\right) \subset V(C)$ and $E\left(C^{*}\right) \subset E(C)$. Given above, we can learn the transition weight $w\left(v_{i}, v_{j}\right)$ to represent the transition likelihood from node to towards treatment relationships $\left(\exists\left(v_{i}, v_{j}\right)\lfloor E(C))\right.$ :

$$
w\left(v_{i}, v_{j}\right)=\left\{\begin{array}{cc}
1+\frac{p\left(v_{i} \rightarrow v_{j} \mid G\left(C^{*}\right)\right)}{p\left(v_{i} \rightarrow v_{j} \mid G(C)\right)} & \text { if }\left(v_{i}, v_{j}\right) \in E\left(C^{*}\right) \\
1 & \text { otherwise }
\end{array}\right.
$$

Where $p\left(v_{i} \rightarrow v_{j} \mid G\left(C^{*}\right)\right)$ and $p\left(v_{i} \rightarrow v_{j} \mid G(C)\right)$ are the transition probabilities from node $v_{i}$ to node $v_{j}$ in $G\left(C^{*}\right)$ and $G(C)$, respectively. Let each chain graph $G(\bullet)$ be a Markov model. Thus the transition probability $p\left(v_{i} \rightarrow v_{j} \mid G(\bullet)\right)$ is computed using maximum likelihood estimation:

$$
p\left(v_{i} \rightarrow v_{j} \mid G(\bullet)\right)=\frac{N v_{i}, v_{j}}{N v_{i}, \bullet}
$$

$N v_{i}, v_{j}$ is the number of times that a transition $v_{i} \rightarrow v_{j}$ is observed in a chain set, and $N v_{i}, \bullet$ is the total number of transitions originated from $v_{i}$ in the chain set.

\section{Predicting novel treatment relationships between drugs} and diseases

For each causal chain $c=\langle d, t, p, g, s>$ in the global chain set $(d C)$, we can estimate its likelihood $L(c)$ based on the pre-computed transition weights in equation (1).

$$
L(c)=\log (w(d, t) \cdot w(t, p) \cdot w(p, g) \cdot w(g, s))
$$

Our prediction of a new indication of drug $d_{x}$ for disease $s_{y}$ is based on the final score $S\left(d_{x}, s_{y}\right)$ between drug $d_{x}$ and disease $s_{y}$, which is the maximal likelihood of all possible chains from $d_{x}$ to $s_{y}$ :

$$
S\left(d_{x}, s_{y}\right)=\max \left(L\left(c_{x, y}\right)\right), \quad c_{x, y} \in\left\{<d_{x}, t_{\bullet}, p_{\bullet}, g_{\bullet}, s_{y}>\right\}
$$

$c_{x, y}$ is a causal chain from drug $d_{x}$ to disease $s_{y}$ among all possible chains $\left.C_{x, y}=\left\{<d_{x}, t, p, g, s_{y}\right\rangle\right\}$. Note that alternatively, $S\left(d_{x} s_{y}\right)$ can also be measured simply by the 
number of successful chains from $d_{x}$ to $s_{y}:\left|C_{x, y}\right|$. As shown below, we used such a method as a baseline for comparing our weighted method.

\section{Results}

Complete and treatment-enriched chain sets

Based on the CauseNet (see Section constructing CauseNet), we constructed a complete causal chain set $C$ including 2,711,440 possible 4-step chains from 979 drugs, to 538 targets, to 207 pathways, to 1,122 downstream genes, to 1,650 diseases, corresponding to 389,945 possible drug-disease associations. A total of 6,268 such associations between 665 drugs and 583 diseases were labelled as known (i.e. found in CTD), resulting in a total of 135,936 chains to the treatment-enriched chain subset $C^{*}$.

Table 1 shows detailed statistics of the complete vs. enriched chain sets and their corresponding graph elements. For each edge in $G(C)$, we calculated its transition weight based on equation 1 (see Section computing transition weights). Furthermore, we computed scores for each of the 389,945 possible drug-disease associations based on the maximal likelihood estimation of causal chains (equation 4) and ranked them accordingly. When treating the known 6,268 associations as the only positive instances, we calculated true positive rate (sensitivity) and false positive rate (1-specificity) of our results at different cut-off ranking scores. As plotted as a ROC curve in Figure 2(A), we obtained a high AUC score of 0.889, which suggests that the 6,289 known (positive) associations were indeed ranked high among all 389,945 pairs. Also in Figure 2(A), we show that our weighted inference method significantly outperformed the baseline method in AUC scores, which shows the value of computing weights for transition between nodes in our CauseNet.

\section{Cross validation of therapeutic effect prediction}

To further evaluate the validity of our method, we conducted a 10 -fold cross validation by withholding $10 \%$ of the known treatment relationships in each fold and removing their connected chains accordingly. Figure 2 (B) shows the results of all ten ROC curves, with the average AUC score of $0.859 \pm 0.006$ with $(\mathrm{CI}=0.95)$ (highlighted in blue). The best tradeoff between sensitivity (0.866) and specificity (0.760) is shown in red, which corresponds to 2.609 in our prediction score. After filtering known ones, 92,057 associations between 964 drugs and 1050 diseases have scores higher than 2.609. Additional File 1 lists the 92,057 predicted associations and all possible causal chains connecting the drug-disease associations via target-and gene-involved pathways.

We compared our method with the similarity-based methods $[9,10]$ which assume that similar drugs are used for similar diseases' treatments. Drug pairwise similarity was measured by chemical 2D structure similarity (SIM_chem), drug target similarity (SIM_target), and linear combination of these two (SIM_combo) respectively. We applied the similarity-based methods to 602 small molecule drugs (with 2D chemical structure) in our CauseNet dataset. As can be seen in Figure 3, our method achieved a higher AUC score (0.866) than using chemical similarity (0.829), target similarity $(0.841)$ or their combination $(0.851)$.

\section{Novel predictions in clinical trials and literature}

We further evaluated our predictions by searching evidence in clinical trials and literature. About $1 / 3$ were found in PubMed [25] (requiring three or more occurrences) and a relatively small percentage of our predictions $(3,202)$ were found in ClinicalTrials.gov [26]. There are several main reasons for more evidence in the literature than in clinical trials: First, some predicted therapeutic uses are still in pre-clinical development and hence have not reached clinical trial stage. For example, we predicted anakinra to treat colorectal neoplasm with a high confidence score of 5.996 .

Table 1 Descriptive statistics of global and treatment enriched chain sets

\begin{tabular}{llcc}
\hline & & Complete chain set $\boldsymbol{C}$ & Enriched chain subset $\boldsymbol{C}^{*}$ \\
\hline \# of chains & & $2,711,440$ & 135,936 \\
\hline Node set & \# of drugs $|\boldsymbol{D}|$ & 979 & 655 \\
\cline { 2 - 4 } \# of targets $|\boldsymbol{T}|$ & 538 & 397 \\
\cline { 2 - 4 } \# of pathways $|\boldsymbol{P}|$ & 207 & 199 \\
\cline { 2 - 4 } \# of genes $|\boldsymbol{G}|$ & 1,122 & 538 \\
\cline { 2 - 4 } \# of diseases $|\boldsymbol{S}|$ & 1,650 & 583 \\
\hline Edge set & $|\boldsymbol{D} \times \boldsymbol{T}|$ & 2,953 & 2,074 \\
& $|\boldsymbol{T} \times \boldsymbol{P}|$ & 2,922 & 2,004 \\
\cline { 2 - 4 } & $|\boldsymbol{P} \times \boldsymbol{G}|$ & 2,772 & 2,179 \\
\cline { 2 - 4 } & $\mid \boldsymbol{G} \times \mathbf{S}$ & 6,496 & 3,954 \\
\hline \# of drug-disease associations & 389,945 & 6,268 \\
\hline
\end{tabular}




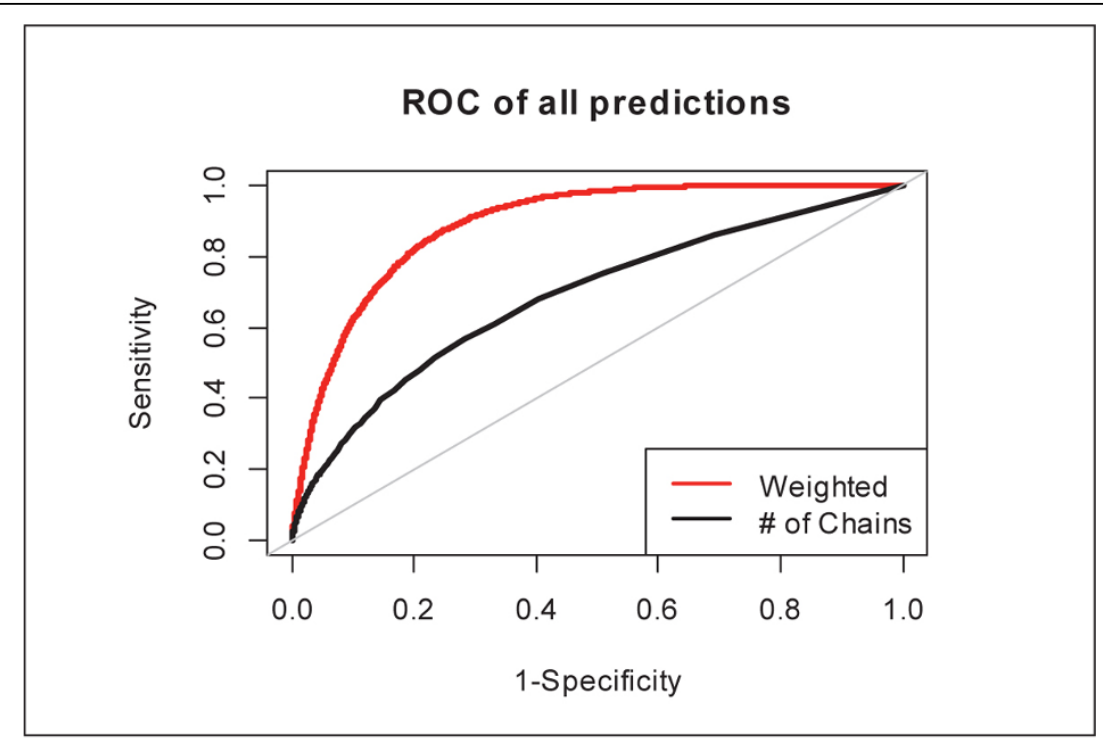

(A)

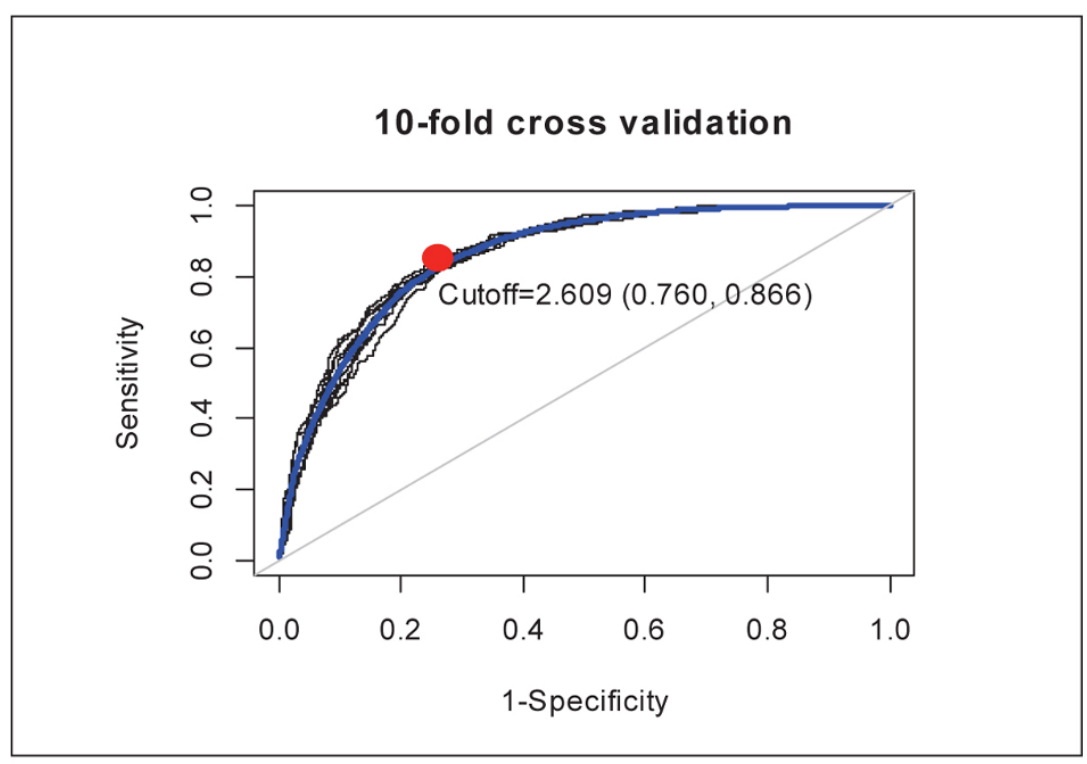

(B)

Figure $\mathbf{2}$ ROC curves of our methods in predicting therapeutic effects.

According to literature evidence [27], anakinra-a drug approved for treating rheumatoid arthritis-was recently found to be able to contribute to growth-inhibition of small tumors in mice with colon carcinoma. Second, clinical trials are not always registered in ClinicalTrails. gov. In our results, some highly scored predictions were found for novel uses of nadroparin-a drug outside of the U.S. market. Some trials have been launched for investigating these new uses in countries outside the
U.S., with their studies reported in literature, but not in ClinicalTrials.gov.

To demonstrate the discriminative power of our prediction scores, we show in Figure 4 that in general the higher the prediction score and more likely the predicted association can be validated in ongoing clinical trial investigations and scientific publications. Hence, we believe such a score can greatly help others to use our prediction results for further investigations. 


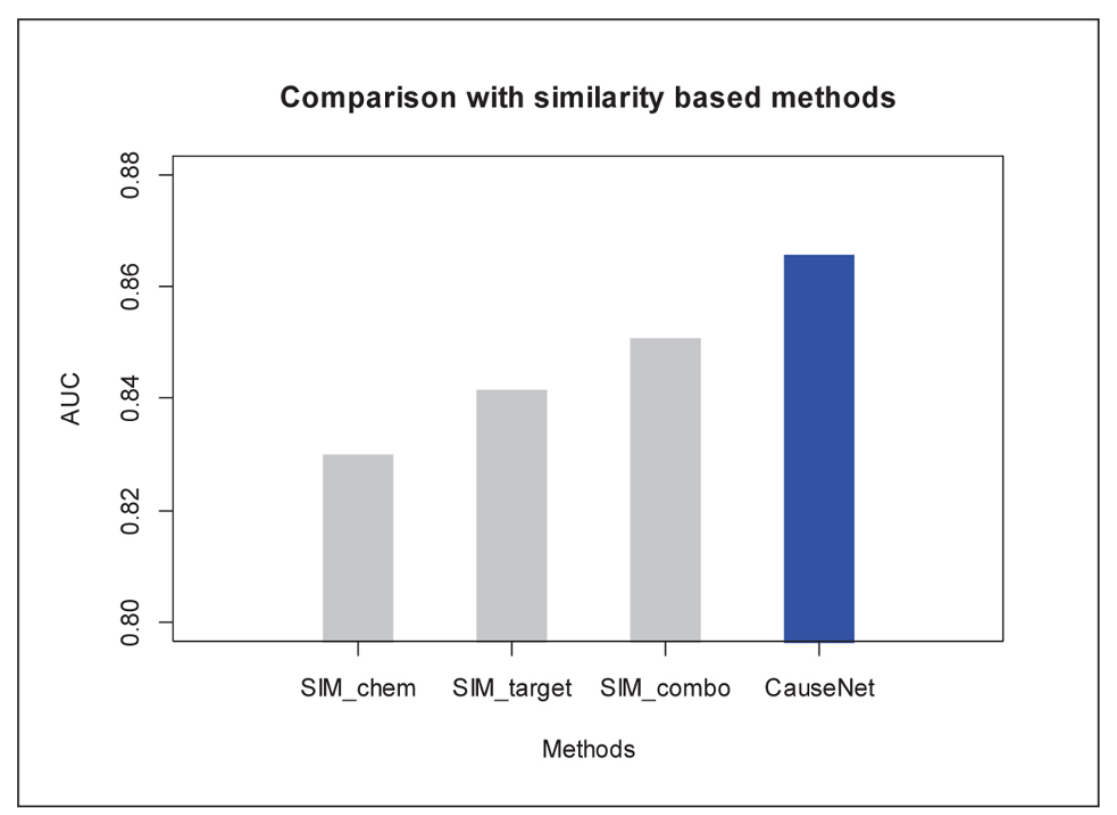

Figure 3 Comparison with similarity-based methods in predicting therapeutic effects.

\section{Investigations of drug repositioning opportunities for Crohn's Disease}

Drug repositioning for poorly treated diseases is a promising strategy in drug discovery today because of the highly unmet need there [5]. In this study, we further explored drug repositioning opportunities for Crohn's disease (CD), a chronic inflammatory condition of the gastrointestinal tract, for which there is no known cure and most treatment options aim to relieve its symptoms such as rectal bleeding and diarrheal [28]. Every year, 10,000 47,000 residents of North America are diagnosed with CD, and as many as 630,000 currently suffer from CD [29]. Epidemiology studies showed incidence of CD is highly influenced by geographic region and family history. Recently, genetic efforts have been made to explain these epidemiologic observations and to understand the underlying pathogenesis from the view of human genomics [30,31]. As a result, multiple CD susceptibility genes have been found such as IL23R, IL6, IL10, NLRP3, FN1, NCF4 and FPR2. These findings could lead to identifying novel therapeutic options for CD.

Figure 5 shows five selected CD drugs predicted by our method for CD and their exemplar causal chains found in our CauseNet. For example, anakinra, an approved rheumatoid arthritis drug, shows a high potential for CD treatment with a score of 5.26 in our method. Further analysis shows that anakinra works by binding receptor IL1R, which may influence multiple pathways like osteoclast differentiation pathway and amoebiasis pathway, affecting CD genes NCF4 and FN1 respectively. Another highly scored drug is nedocromil (score $=4.00$ ), a drug approved for treating allergic conjunctivitis and asthma. Our method shows its potential therapeutic use in CD through acting on multiple targets HSP90AA1 and FPR1, affecting multiple pathways NOD-like receptor signaling pathway and staphylococcus aureus infection pathway, and further affecting multiple CD mechanism genes IL6, TNF, NLRP3, NOD2, FPR2 and IL10. This comprehensive evidence would greatly help experts generate hypotheses on the therapeutic values of these CD drug candidates which are worth further experimenting. We find that two drugs shown in Figure 5, adalimumab and prednisolone, have also been previously studied for CD [32,33].

\section{Discussion}

In this study, we propose a new computational drug repositioning approach by using causal chains in drug-disease networks (see Figure 1). Our method has the following important characteristics:

First, it provides a broad and semantic view of molecular causality between drugs and diseases. Unlike the traditional 'one drug-one target-one disease' model, we put all causality relationships between drugs and disease in a network view with five distinct layers. In the CauseNet construction, we integrated different types of data and semantic relationships between them from widely recognized and expert-curated resources. For example, when integrating pathway data, we focused on specific direction (downstream) and specific semantics (transcriptional response) relationships in an interested pathway by taking advantage of recent progress in pathway curation and standardization $[21,34]$. The resulting CauseNet laid down 


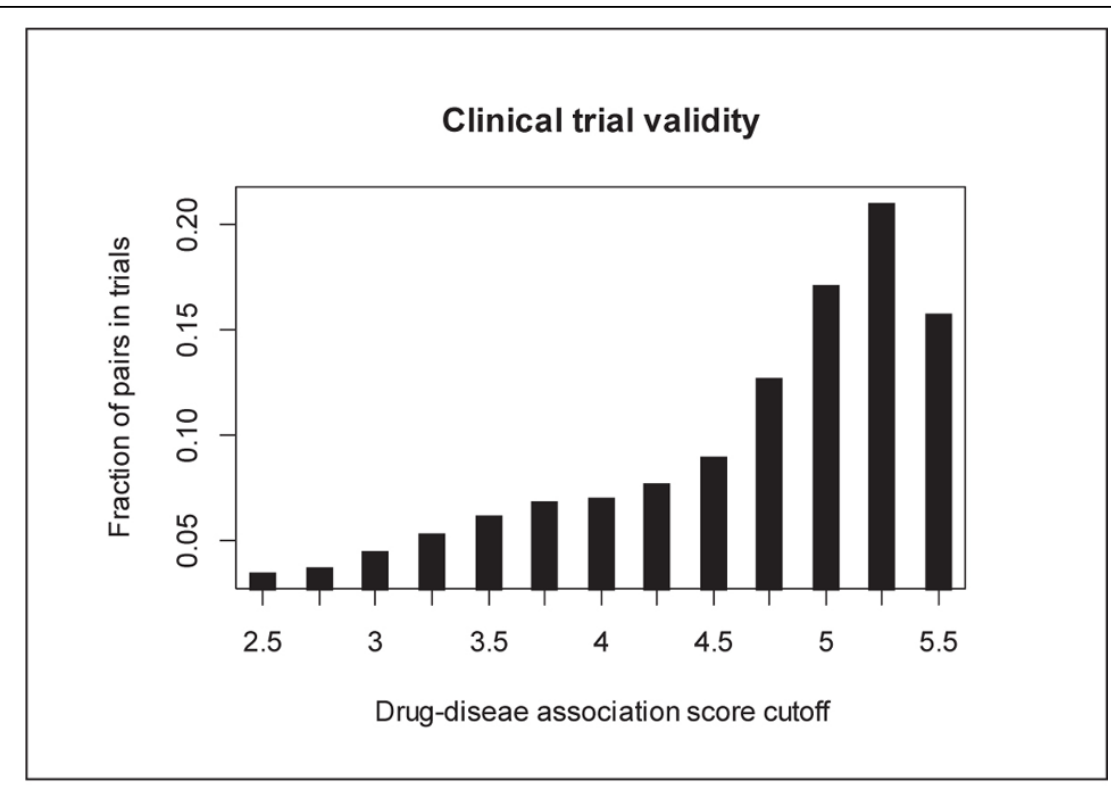

(A)

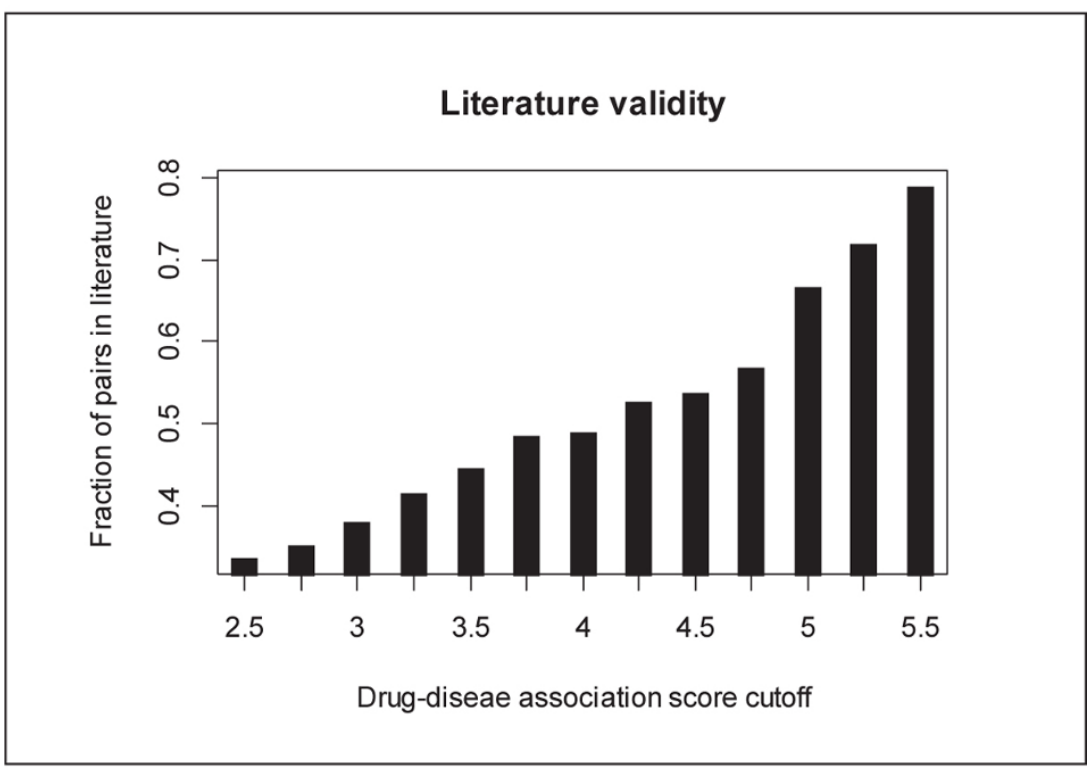

(B)

Figure 4 Clinical trial and literature validity of novel drug-disease association predictions.

a key foundation for further drug-disease relationship prediction.

Second, not only does our method find novel drugdisease treatment associations, but also scores and ranks each prediction accordingly. As shown in the crossvalidation experiment, our method is able to rank true associations generally at the top positions. Moreover, those highly scored drug-disease prediction results are found significantly enriched in clinical trials and biomedical literature. Hence, we believe that our weighted inference method is able to prioritize prediction results for further exploring drug repositioning opportunities.

Third, instead of being a black box, our method provides detailed and comprehensive molecular evidence supporting each prediction. As shown in the case study with Crohn's disease, the accompanying pathway evidence can 


\begin{tabular}{|c|c|}
\hline Drug & $\begin{array}{c}\text { Selected Causal Chains Supporting This Prediction } \\
\text { (D Drug; T Target; P Pathway; G Downstream Gene; } \mathrm{S} \text { Disease) }\end{array}$ \\
\hline \multirow{2}{*}{$\begin{array}{c}\text { Anakinra } \\
(\text { score }=5.29)\end{array}$} & D Anakinra $\rightarrow$ T IL1R $\rightarrow \mathrm{P}$ Osteoclast differentiation $\rightarrow \mathrm{G}$ NCF4 $\rightarrow \mathrm{S}$ CD \\
\hline & D Anakinra $\rightarrow$ T IL1R $\rightarrow \mathrm{P}$ Amoebiasis $\rightarrow$ G FN1 $\rightarrow \mathrm{S}$ CD \\
\hline \multirow{2}{*}{$\begin{array}{c}\text { Rifabutin } \\
(\text { score }=4.30)\end{array}$} & $\begin{array}{l}\text { D Rifabutin } \rightarrow \text { T HSP90AA1 } \rightarrow \text { P NOD-like receptor signaling pathway } \rightarrow \\
\text { G IL6, TNF, NLRP3, NOD2 } \rightarrow \mathrm{S} \text { CD }\end{array}$ \\
\hline & $\begin{array}{l}\text { D Rifabutin } \rightarrow \text { T HSP90AA1 } \rightarrow \text { P Glucocorticoid receptor regulatory network } \rightarrow \\
\text { G IL6 } \rightarrow \mathrm{S} \text { CD }\end{array}$ \\
\hline \multirow{2}{*}{$\begin{array}{l}\text { Nedocromil } \\
(\text { score }=4.00)\end{array}$} & $\begin{array}{l}\text { D Nedocromil } \rightarrow \text { T HSP90AA1 } \rightarrow \text { P NOD-like receptor signaling pathway } \rightarrow \\
\text { G IL6, TNF, NLRP3, NOD2 } \rightarrow \mathrm{S} \text { CD }\end{array}$ \\
\hline & $\begin{array}{l}\text { D Nedocromil } \rightarrow \text { T FPR1 } \rightarrow \text { P Staphylococcus aureus infection } \rightarrow \\
\text { G FPR2, IL10 } \rightarrow \text { S CD }\end{array}$ \\
\hline \multirow{2}{*}{$\begin{array}{l}\text { Adalimumab } \\
(\text { score }=3.64)\end{array}$} & $\begin{array}{l}\text { D Adalimumab } \rightarrow \mathrm{T} \text { TNF, FCGR1A } \rightarrow \mathrm{P} \text { Tuberculosis } \rightarrow \text { G TNF, IL6, IL10, IL23R } \\
\rightarrow \mathrm{S} \text { CD }\end{array}$ \\
\hline & $\begin{array}{l}\text { D Adalimumab } \rightarrow \text { T FCGR1A, C1R, C1QA, C1S } \rightarrow \text { P Staphylococcus aureus } \\
\text { infection } \rightarrow \text { G FPR2, IL10 } \rightarrow \mathrm{S} \text { CD }\end{array}$ \\
\hline \multirow{2}{*}{$\begin{array}{l}\text { Prednisolone } \\
(\text { score }=2.96)\end{array}$} & $\begin{array}{l}\text { D Prednisolone } \rightarrow \mathrm{T} \text { NR3C1 } \rightarrow \text { P Glucocorticoid receptor regulatory network } \rightarrow \\
\text { G IL6 } \rightarrow \mathrm{S} \text { CD }\end{array}$ \\
\hline & $\begin{array}{l}\text { D Prednisolone } \rightarrow \text { T NR3C1 } \rightarrow \text { P AP-1 transcription factor network } \rightarrow \\
\text { G IL6, IL10 } \rightarrow \mathrm{S} \text { CD }\end{array}$ \\
\hline
\end{tabular}

Figure 5 Potential drugs for Crohn's Disease (CD) treatment.

support further human investigation. More importantly, such comprehensive pathway information could reveal unknown linkages between drugs and disease and help hypothesis generation on novel drug re-uses.

Lastly, our prediction results cover a wide range of diseases and drugs. For drugs, our repositioning results consist of both small molecule drugs (e.g., rifabutin) and big molecules (e.g., adalimumab), thus lifting the limitations of those methods that rely on 2D chemical structures or gene expression profiles of small molecules [9-14]. In addition, our method can identify drugs for a disease with no current treatments, making it different from similaritybased methods where predictions are always based on known uses of other drugs.

Like other knowledge-based methods, our approach relies on existing knowledge of drug-target, target-pathway, pathway-downstream gene, gene-disease, and drugdisease relationships. Despite increasing efforts in data curation and standardization, at present such information is still incomplete, thus limiting the prediction power of our method. For example, we extracted 1,239 targetinvolved pathways, but merely 209 of which contain transcriptional response relationships. Combining gene expression with pathway analysis to predict downstream genes is a hopeful strategy to help break the bottleneck [35]. We plan to investigate this issue in future work.

\section{Conclusions}

In this study, we successfully developed a computational drug repositioning method using pathway-based causal inference. Unlike the traditional 'one drug-one target-one disease' causal model, we systematically considered all possible causal chains connecting drugs to diseases via target- and gene-involved pathways. More specifically, we built a multi-layer causal network (CauseNet) consisting of chains from drugs to disease by integrating heterogeneous expert-curated biological resources in public domain. The transition likelihood of each causal edge in the CauseNet was estimated by learning known drug-disease treatment relationships. Furthermore, we predicated novel drug indications using maximum likelihood estimation of causal chains between drugs and diseases. In cross-validation experiments, our method achieved AUC score of $0.859 \pm 0.006$ with best tradeoff sensitivity = 0.866 and specificity $=0.760$. When compared with a control group of drug uses, our drug repositioning results were found to be significantly enriched in both the biomedical literature and clinical trials. Additionally, in the Crohn's Disease case study, we demonstrated our method would provide more comprehensive evidence showing how drugs connect to diseases via pathways. We believe our method would greatly help experts generate hypotheses in drug discovery. 


\section{Additional material}

Additional file 1: Predicted drug-disease associations. lists the 92,057 predicted associations and all possible causal chains connecting the drug-disease associations via target-and gene-involved pathways

\section{Competing interests}

The authors declare that they have no competing interests.

\section{Authors' contributions}

$J \mathrm{~L}$ and $\mathrm{ZL}$ conceived the whole study, participated in its design, analyzed the results and wrote the manuscript. $J$ collected the data, implemented the methods and performed the experiments. All authors read and approved the final manuscript.

\section{Acknowledgements}

This research was supported by the Intramural Research Program of the National Institutes of Health, the National Key Technology R\&D Program of China (Grant No. 2013BAl06B01) and the Peking Union Medical College Youth Fund. The authors would like to thank the Pathway Commons team for discussing meaning use of their data, and Bethany Harris for proofreading our manuscript.

\section{Declarations}

Publication of this article was funded by the Intramural Research Program of the National Institutes of Health.

This article has been published as part of BMC Bioinformatics Volume 14 Supplement 16, 2013: Twelfth International Conference on Bioinformatics (InCoB2013): Bioinformatics. The full contents of the supplement are available online at http://www.biomedcentral.com/bmcbioinformatics/ supplements/14/S16.

\section{Authors' details}

${ }^{1}$ Institute of Medical Information, Chinese Academy of Medical Sciences, Beijing, China. ${ }^{2}$ National Center for Biotechnology Information (NCBI), National Institutes of Health, Bethesda, USA.

Published: 22 October 2013

\section{References}

1. Kim DH, Sim T: Chemical kinomics: a powerful strategy for target deconvolution. BMB Rep 2010, 43(11):711-719.

2. Roemer T, Davies J, Giaever G, Nislow C: Bugs, drugs and chemical genomics. Nat Chem Biol 2012, 8(1):46-56.

3. Wang Y, Xiao J, Suzek TO, Zhang J, Wang J, Bryant SH: PubChem: a public information system for analyzing bioactivities of small molecules. Nucleic Acids Res 2009, 37(Web Server):W623-633.

4. Gaulton A, Bellis $\amalg$, Bento AP, Chambers J, Davies M, Hersey A, Light $Y$, McGlinchey S, Michalovich D, Al-Lazikani B, et al: ChEMBL: a large-scale bioactivity database for drug discovery. Nucleic Acids Res 2012, 40(Database):D1100-1107.

5. Scannell JW, Blanckley A, Boldon H, Warrington B: Diagnosing the decline in pharmaceutical R\&D efficiency. Nat Rev Drug Discov 2012, 11(3):191-200.

6. Ashburn $\Pi$, Thor KB: Drug repositioning: identifying and developing new uses for existing drugs. Nat Rev Drug Discov 2004, 3(8):673-683.

7. Dudley JT, Schadt E, Sirota M, Butte AJ, Ashley E: Drug discovery in a multidimensional world: systems, patterns, and networks. J Cardiovasc Transl Res 2010, 3(5):438-447.

8. Schadt EE, Friend SH, Shaywitz DA: A network view of disease and compound screening. Nat Rev Drug Discov 2009, 8(4):286-295.

9. Keiser MJ, Setola V, Irwin JJ, Laggner C, Abbas Al, Hufeisen SJ, Jensen NH, Kuijer MB, Matos RC, Tran TB, et al: Predicting new molecular targets for known drugs. Nature 2009, 462(7270):175-181

10. Li J, Lu Z: A New Method for Computational Drug Repositioning Using Drug Pairwise Similarity. Proceedings of The IEEE International Conference on Bioinformatics and Biomedicine (BIBM) Philadelphia, USA; 2012
11. Iorio F, Bosotti R, Scacheri E, Belcastro V, Mithbaokar P, Ferriero R, Murino L, Tagliaferri R, Brunetti-Pierri N, Isacchi A, et al: Discovery of drug mode of action and drug repositioning from transcriptional responses. Proc Natl Acad Sci USA 2010, 107(33):14621-14626.

12. $\mathrm{Hu} \mathrm{G}$, Agarwal P: Human disease-drug network based on genomic expression profiles. PLoS One 2009, 4(8):e6536.

13. Sirota M, Dudley JT, Kim J, Chiang AP, Morgan AA, Sweet-Cordero A, Sage J, Butte AJ: Discovery and preclinical validation of drug indications using compendia of public gene expression data. Sci Transl Med 2011, 3(96):96ra77.

14. Shigemizu D, Hu Z, Hung JH, Huang CL, Wang Y, DeLisi C: Using functional signatures to identify repositioned drugs for breast, myelogenous leukemia and prostate cancer. PLOS Comput Biol 2012, 8(2): e1002347.

15. Li J, Zhu X, Chen JY: Building disease-specific drug-protein connectivity maps from molecular interaction networks and PubMed abstracts. PLoS Comput Biol 2009, 5(7):e1000450.

16. Li Y, Agarwal P: A pathway-based view of human diseases and disease relationships. PLoS One 2009, 4(2):e4346.

17. Strittmatter WJ: Medicine. Old drug, new hope for Alzheimer's disease. Science 2012, 335(6075):1447-1448.

18. Sivachenko A, Kalinin A, Yuryev A: Pathway analysis for design of promiscuous drugs and selective drug mixtures. Curr Drug Discov Technol 2006, 3(4):269-277

19. Cramer PE, Cirrito JR, Wesson DW, Lee CY, Karlo JC, Zinn AE, Casali BT, Restivo JL, Goebel WD, James MJ, et al: ApoE-directed therapeutics rapidly clear beta-amyloid and reverse deficits in AD mouse models. Science 2012, 335(6075):1503-1506.

20. Kotelnikova E, Yuryev A, Mazo I, Daraselia N: Computational approaches for drug repositioning and combination therapy design. J Bioinform Comput Biol 2010, 8(3):593-606.

21. Cerami EG, Gross BE, Demir E, Rodchenkov I, Babur O, Anwar N, Schultz N, Bader GD, Sander C: Pathway Commons, a web resource for biological pathway data. Nucleic Acids Res 2011, 39(Database):D685-690.

22. Wishart DS, Knox C, Guo AC, Cheng D, Shrivastava S, Tzur D, Gautam B, Hassanali M: DrugBank: a knowledgebase for drugs, drug actions and drug targets. Nucleic Acids Res 2008, 36(Database):D901-906.

23. Kanehisa M, Goto S, Furumichi M, Tanabe M, Hirakawa M: KEGG for representation and analysis of molecular networks involving diseases and drugs. Nucleic Acids Res 2010, 38(Database):D355-360.

24. Davis AP, Murphy CG, Saraceni-Richards CA, Rosenstein MC, Wiegers TC, Mattingly CJ: Comparative Toxicogenomics Database: a knowledgebase and discovery tool for chemical-gene-disease networks. Nucleic Acids Res 2009, 37(Database):D786-792.

25. PubMed ${ }^{\mathbb{R}}$. [http://www.ncbi.nlm.nih.gov/pubmed/].

26. ClinicalTrials.gov. [http://clinicaltrials.gov/].

27. Harnack $U$, Johnen $H$, Pecher G: IL-1 receptor antagonist anakinra enhances tumour growth inhibition in mice receiving peptide vaccination and beta-(1-3),(1-6)-D-glucan. Anticancer Res 2010, 30(10):3959-3965.

28. Crohn's Disease. [http://digestive.niddk.nih.gov/ddiseases/pubs/crohns/]

29. Loftus EV Jr: Clinical epidemiology of inflammatory bowel disease: Incidence, prevalence, and environmental influences. Gastroenterology 2004, 126(6):1504-1517.

30. Rioux JD, Xavier RJ, Taylor KD, Silverberg MS, Goyette P, Huett A, Green T, Kuballa P, Barmada MM, Datta LW, et al: Genome-wide association study identifies new susceptibility loci for Crohn disease and implicates autophagy in disease pathogenesis. Nat Genet 2007, 39(5):596-604

31. Kenny EE, Pe'er I, Karban A, Ozelius L, Mitchell AA, Ng SM, Erazo M, Ostrer H, Abraham C, Abreu MT, et al: A genome-wide scan of Ashkenazi Jewish Crohn's disease suggests novel susceptibility loci. PLoS Genet 2012, 8(3):e1002559.

32. Dudley JT, Sirota M, Shenoy M, Pai RK, Roedder S, Chiang AP, Morgan AA, Sarwal MM, Pasricha PJ, Butte AJ: Computational repositioning of the anticonvulsant topiramate for inflammatory bowel disease. Sci Trans/ Med 2011, 3(96):96ra76.

33. Adalimumab in FDA orphan drug product designation database. [http://www.accessdata.fda.gov/scripts/opdlisting/oopd/OOPD_Results_2 cfm?Index_Number=230306]. 
34. Demir E, Cary MP, Paley S, Fukuda K, Lemer C, Vastrik I, Wu G,

D'Eustachio P, Schaefer C, Luciano J, et al: The BioPAX community

standard for pathway data sharing. Nat Biotechnol 2010, 28(9):935-942.

35. Babur O, Demir E, Gonen M, Sander C, Dogrusoz U: Discovering

modulators of gene expression. Nucleic Acids Res 2010, 38(17):5648-5656.

doi:10.1186/1471-2105-14-S16-S3

Cite this article as: $\mathrm{Li}$ and $\mathrm{Lu}$ : Pathway-based drug repositioning using causal inference. BMC Bioinformatics 2013 14(Suppl 16):S3.

Submit your next manuscript to BioMed Central and take full advantage of:

- Convenient online submission

- Thorough peer review

- No space constraints or color figure charges

- Immediate publication on acceptance

- Inclusion in PubMed, CAS, Scopus and Google Scholar

- Research which is freely available for redistribution

Submit your manuscript at www.biomedcentral.com/submit 